\title{
Simulation of vortex sound using the viscous/acoustic splitting approach
}

\author{
Zheng, Ting H.; Tang, Shiu K.; Shen, Wen Zhong
}

Published in:

Transactions of the Canadian Society for Mechanical Engineering

Publication date:

2011

Document Version

Publisher's PDF, also known as Version of record

Link back to DTU Orbit

Citation (APA):

Zheng, T. H., Tang, S. K., \& Shen, W. Z. (2011). Simulation of vortex sound using the viscous/acoustic splitting approach. Transactions of the Canadian Society for Mechanical Engineering, 35(1), 39-56.

http://www.tcsme.org/Papers/Vol35/Vol35No1Paper3.pdf

\section{General rights}

Copyright and moral rights for the publications made accessible in the public portal are retained by the authors and/or other copyright owners and it is a condition of accessing publications that users recognise and abide by the legal requirements associated with these rights.

- Users may download and print one copy of any publication from the public portal for the purpose of private study or research.

- You may not further distribute the material or use it for any profit-making activity or commercial gain

- You may freely distribute the URL identifying the publication in the public portal

If you believe that this document breaches copyright please contact us providing details, and we will remove access to the work immediately and investigate your claim. 


\title{
SIMULATION OF VORTEX SOUND USING THE VISCOUS/ACOUSTIC SPLITTING APPROACH
}

\author{
Ting H. Zheng ${ }^{1}$, Shiu K. Tang ${ }^{2}$, Wen Z. Shen ${ }^{3}$ \\ ${ }^{1}$ Department of Applied Mechanics, Sichuan University, Chengdu, P.R. China, 610065 \\ ${ }^{2}$ Department of Building Service Engineering, The Hong Kong Polytechnic University, Hong Kong, P.R. China \\ 3 Department of Engineering, Technical University of Denmark, Denmark \\ E-mail: besktang@polyu.edu.hk
}

Received March 2010, Accepted April 2010

No. 10-CSME-14, E.I.C. Accession 3177

\begin{abstract}
A numerical viscous/acoustic splitting approach for the calculation of an acoustic field is applied to study the sound generation by a pair of spinning vortices and by the unsteady interaction between an inviscid vortex and a finite length flexible boundary. Based on the unsteady hydrodynamic information from the known incompressible flow field, the perturbed compressible acoustic terms are calculated and compared with analytical solutions. Results suggest that the present numerical approach produces results which are in good agreement with the analytical solutions. The present investigation verifies the applicability of the viscous/ acoustic approach to flow structure-acoustic interaction.
\end{abstract}

Keywords: computational aeroacoustics; vortex sound; viscous/acoustic approach.

\section{SIMULATIONS NUMÉRIQUES DE PRODUCTION SONORE PAR DES ÉCOULEMENTS TOURBILLONNAIRES À L'AIDE DE DÉCOMPOSITION VORTICITE/POTENTIEL}

\section{RÉSUMÉ}

Une méthode de décomposition des écoulements en composantes acoustiques et vorticelles est appliquée au problème de la génération sonore pour un couple de deux vortex tournants, ainsi que le bruit d'interaction entre un vortex idéal et une surface flexible. Les contributions linéaires acoustiques sont évaluées par comparaison avec la solution analytique connue pour l'écoulement idéal potentiel incompressible. Le champ sonore résultant est comparé à une solution analytique. Les résultats sont satisfaisants, et suggèrent des applications possibles pour les interactions fluides-structures.

Mots-clés : calcul aéroacoustique, son tourbillon, méthode de décomposition des écoulements. 


\section{INTRODUCTION}

Aeroacoustics, which is the science of noise generation by airflows and its propagation, is a relatively young discipline compared to other more classical fields of mechanics. Typically in aeroacoustics, the solutions can be grouped into the frequency-domain solution and the natural variables $(x, t)$ solution. Within the latter, the different approaches in the analysis can be categorized into three groups.

The first group makes use of the acoustic analogy. The most renowned acoustic analogy is due to Lighthill [1]. He rearranged the mass and momentum equations to obtain an inhomogeneous wave equation which describes the generation and propagation of an acoustic wave in a medium at rest. Ffowcs Williams and Hawkings [2] generalized the Lighthill's acoustic analogy to include the effects of surfaces in arbitrary motions. Powell [3] and Möhring [4] put forward the vortex sound theory for predicting the sound from two and threedimensional compact vortical flows. This group of approach can compute the noise directivity in an economical way because the flow in the far field is actually uniform. However, all of the acoustic analogies are based on a variety of assumptions such as compact source and low Mach number. Acoustically compact sources (including a vortex) mean that the size of the acoustic source is much smaller than the wavelength of the acoustic waves generated. In fact, in many practical cases, the latter is comparable to the former. The advantage of numerical simulation is that this compact source assumption is not needed.

The second approach makes use of direct numerical simulation (DNS), where both the fluid motion and the generated sound are directly computed by means of the Navier-Stokes equations. One of the advantages of DNS is its capability to compute the generation and propagation processes without suffering from restrictions such as low Mach number, high Reynolds number and compactness of the source region in principle. DNS methods are specially suited to model broadband noise generated by turbulence. However, it is difficult to distinguish pressure fluctuations from the sound generated as the acoustic perturbations are typically at least 10 times weaker than the corresponding hydrodynamic perturbations [5]. Also, DNS requires tremendous computational resources especially when the flow Mach number is low and it is difficult to compute propagation over long distances. Therefore, this approach is preferable for the study of aeroacoustics at a relatively higher Mach number condition [6]. Some authors neglect the viscosity and flow turbulence and solve the Euler equations for short-time, high-speed sound-generating phenomena such as shock wave-vortex interaction (for instance, Inoue and Hatakeyama [7]).

The third approach uses the hydrodynamic/acoustic splitting method which decomposed the flow field into incompressible hydrodynamic and compressible perturbation equations (for instance, Hardin and Pope [8]). This splitting method has further been modified by Shen and Sørensen [9]. Bogey et al. [10] computed the sound radiated by unsteady fluid motions using an acoustic analogy based on the linear Euler equations forced by aerodynamic source terms. Seo and Moon [11] revised this hydrodynamic/acoustic splitting method through a consideration of the perturbed vorticity transport equation. More recently, Ewert and Schröder [12] derived several source term formulations to allow the acoustic simulation based on incompressible and compressible flow solutions. This approach does not allow acoustic backscattering into the flow solution. It makes possible the computation of aeroacoustic noise generation and propagation in non-uniform unsteady viscous flows in complex domains. This poses substantial advantage over the first approach. Also, compared with the acoustic analogy theories, the sound strength is obtained directly in this approach so that both sound radiation and scattering can be 
accounted for simultaneously [6]. On the other hand, the computing cost required is much lower than that for the DNS solution of the time-dependent subsonic flow field.

The turbulent air flow inside air conditioning ductwork induces pressure fluctuations on the duct walls, resulting in the vibration of the latter which in turn radiates sound to both the duct interior and the external environment. Understanding the flow structure-acoustics interaction therefore is important for improved duct noise control design. Tang et al. [13] developed a theoretical model to investigate the sound generated by the unsteady interactions between a vortex and a finite length flexible boundary in an otherwise rigid wall at low Mach numbers. It is concluded that the time fluctuating volumetric flow rate induced by the flexible boundary vibration and the vortex acceleration are two major sources of sound.

However, real duct flows are turbulent and analytical solution is hard to find for this kind of aeroacoustics problem while numerical solution will make it possible to investigate the turbulence-structure interaction. A turbulence modeling technique can be used to recover the turbulent activities and therefore provide the hydrodynamic terms needed in the acoustic/viscous splitting method. The acoustic radiation can then be obtained directly through numerical means.

In this paper, the viscous/acoustic splitting method is applied to calculate the sound generation by the spinning vortices [14-15] and by the vortex induced flexible wall vibration [13]. The computed results are compared with the analytical solutions and the performance of this viscous/acoustic splitting method is examined. The major aim of this paper is to verify the applicability of the numerical technique to the simplified models of flow noise radiation with and without a nearby solid structure. It is hoped that the present results will provide clues for the modeling of more complicated aeroacoustic problems.

\section{NUMERICAL PROCEDURES}

\subsection{General Formulations}

According to Shen and Sørensen [5], the compressible solution can be decomposed as:

$$
u=U+u^{\prime}, v=V+v^{\prime}, p=P+p^{\prime}, \rho=\rho_{o}+\rho^{\prime},
$$

where $U, V, P$ and $\rho_{o}$ are the background mean flow longitudinal and transverse velocities, fluid pressure and density respectively. They can be obtained analytically or numerically using loworder schemes of computational fluid dynamics, while $u^{\prime}, v^{\prime}, p^{\prime}$ and $\rho^{\prime}$ are corresponding acoustic disturbances obtained from the numerical solutions using high-order schemes in space and time to precisely capture the sound pressure.

Substituting the above equations into the compressible Navier-Stokes equations, neglecting the viscous terms and the higher order perturbations, and subtracting the incompressible conservation equations, the set of governing equations for the two-dimensional acoustic fields can be expressed as:

$$
\begin{aligned}
& \frac{\partial \rho^{\prime}}{\partial t}+\frac{\partial f_{i}}{\partial x_{i}}=0 \\
& \frac{\partial f_{i}}{\partial t}+\frac{\partial}{\partial x_{j}}\left[p^{\prime} \delta_{i j}+f_{i}\left(U_{j}+u_{j}^{\prime}\right)+\rho_{o} U_{i} u_{j}^{\prime}\right]=0 \\
& \frac{\partial p^{\prime}}{\partial t}-c^{2} \frac{\partial \rho^{\prime}}{\partial t}=-\frac{\partial P}{\partial t} .
\end{aligned}
$$


where

$$
f_{i}=\rho u_{i}^{\prime}+\rho^{\prime} U_{i}
$$

$c$, the ambient speed of sound, is obtained from the equation $c^{2}=\gamma p / \rho=\gamma\left(P+p^{\prime}\right) /\left(\rho_{o}+\rho^{\prime}\right)$ and $\gamma$ is the specific heat ratio. Details of the formulation of Eq. (2) are given in the Appendix. It should be noted that the only acoustic source coming from the incompressible solution is the instantaneous pressure and the acoustic calculations can thus be started at any time during the incompressible computation. The initial conditions are

$$
\rho^{\prime}=0, u_{i}^{\prime}=0 \text { and } p^{\prime}=p_{o}-P
$$

where $p_{o}$ is the ambient air pressure.

\subsection{Numerical Discretization}

The acoustic waves are non-dispersive and non-dissipative during their propagation. They are particularly susceptible to numerical dispersion and dissipation. Numerical dispersion distorts the phase between various waves and numerical dissipation reduces the gradients in the solutions [16]. One straightforward way to overcome these problems is to use a very fine grid together with a standard low-order method. However, this approach is not truly feasible because of the dramatic increase of the computation time and memory. To accurately resolve the propagation of acoustic waves, finite difference and time-marching schemes that have low numerical dissipation and can accurately present the dispersion relation for the inviscid equations are required [7].

In this study, the forth-order-accurate central-difference compact scheme [17] that has low dissipation and near spectral representation of the dispersion relationship is chosen for the numerical approximation of the spatial derivatives in Eq. (2):

$$
\frac{1}{4} \Omega_{i-1}^{\prime}+\Omega_{i}^{\prime}+\frac{1}{4} \Omega_{i+1}^{\prime}=\frac{3}{4 \Delta x}\left(\Omega_{i+1}-\Omega_{i-1}\right),
$$

where $\Omega=\rho^{\prime}, f_{1}{ }^{\prime}, f_{2}{ }^{\prime}, p^{\prime}$, and $\Delta x$ is the grid size. At the boundary of computational domain, a third-order-accurate compact scheme biased toward the interior nodes is used [17]:

$$
\begin{aligned}
& \Omega_{1}^{\prime}+2 \Omega_{2}^{\prime}=\frac{1}{\Delta x}\left(-\frac{5}{2} \Omega_{1}+2 \Omega_{2}+\frac{3}{2} \Omega_{3}\right), \\
& \Omega_{n}^{\prime}+2 \Omega_{n-1}^{\prime}=\frac{1}{\Delta x}\left(-\frac{5}{2} \Omega_{n}+2 \Omega_{n-1}+\frac{3}{2} \Omega_{n-2}\right)
\end{aligned}
$$

The centered nondissipative compact scheme is desirable for the computation of linear wave propagation. However, the inherent lack of numerical dissipation may also result in spurious numerical oscillations and instability. To overcome its unrestricted growth of spurious perturbations, the tenth order filter scheme [15] is employed as such scheme does not amplify any waves, preserves constant functions and completely eliminates the odd-even mode when uniform meshes are taken. 
If a solution vector is denoted by $\lambda$, the filtered values $\hat{\lambda}$ satisfy,

$$
\alpha_{f} \hat{\lambda}_{i-1}+\hat{\lambda}_{i}+\alpha_{f} \hat{\lambda}_{i+1}=\sum_{n=0}^{N} \frac{a_{n}}{2}\left(\lambda_{i+n}+\lambda_{i-n}\right)
$$

where $\alpha_{f}$ is the filter coefficient and $-0.5<\alpha_{f} \leq 0.5$. A higher value of $\alpha_{f}$ corresponds to a less dissipative filter and there will be no filtering effect for $\alpha_{f}=0.5$. In this paper, $\alpha_{f}=0.45$. The $N+1$ coefficients $a_{0}, a_{1}, \cdots a_{N}$, are functions of $\alpha_{f}$. With a proper choice of the coefficients, Eq. (7) provides a $2 N$ th-order formula on a $2 N+1$ point stencil. In this study, the tenth order filtering scheme are employed and the corresponding coefficients of $a_{n}$ can be found in Visbal and Gaitonde [18].

Since the compact schemes near boundaries are not symmetrical and they contain dissipation factors already, no filtering scheme is needed at the boundary point 0 and $I L-1$. At a nearboundary point, $i$, the one-sided filter formula is given by

$$
\alpha_{f} \hat{\lambda}_{i-1}+\hat{\lambda}_{i}+\alpha_{f} \hat{\lambda}_{i+1}=\sum_{n=0}^{10} a_{n, i} \lambda_{n}, i \in\{1, \cdots 4\}
$$

and

$$
\alpha_{f} \hat{\lambda}_{i-1}+\hat{\lambda}_{i}+\alpha_{f} \hat{\lambda}_{i+1}=\sum_{n=0}^{10} a_{I L-n, i} \hat{\lambda}_{I L-n}, i \in\{I L-5, \cdots I L-1\}
$$

More information about the coefficients of $a_{n}$ can be found in Gaitonde and Visbal [19].

The explicit fourth-order Runge-kutta time advancement scheme proposed by Williamson [20] and implemented by Wilson et al. [21] is adopted in the present study as it gives low amplitude and phase errors of traveling wave solutions. To simplify the discussion, consider the following convective wave equation:

$$
\frac{\partial \Omega}{\partial t}+\frac{\partial \Omega}{\partial x}+\frac{\partial \Omega}{\partial y}=0
$$

The equation is advanced from time level $n$ to $n+1$ in $Q$ sub-stages. The advancement from sub-stage $M$ to $M+1$ is defined by

$$
\Omega^{M+1}=\Omega^{M}+b^{M+1} H^{M} \Delta t,
$$

where $M=1, \ldots, 5$ is the particular stage number, $\Delta t$ is the time step, $b^{M}$ is a coefficient that can be found in Williamson [20] and $\Omega^{M}$ represents the $\Omega$ value at the $M^{\text {th }}$ sub-stage. $H^{M}$ is the sum of all the right-hand-side terms in Eq. (2). The accumulation from the previous sub-stage or from the initial conditions at $t=0$ is

$$
H^{M}=\frac{\partial \Omega^{M}}{\partial x}+\frac{\partial \Omega^{M}}{\partial y}+a^{M} H^{M-1},
$$


where $a^{M}$ is another coefficient that can be found in Williamson [20]. The low-storage requirement is accomplished by continuously overwriting the storage location for the time derivatives and unknown variables at each sub-stage:

$$
a^{M} H^{M-1} \rightarrow H^{M} \text { and } \Omega^{M}+b^{M+1} H^{M} \Delta t \rightarrow \Omega^{M+1}
$$

where $\rightarrow$ indicates that the storage locations $H^{M-1}$ and $\Omega^{M}$ are overwritten by $H^{M}$ and $\Omega^{M+1}$ at each time step respectively.

\subsection{Boundary Conditions}

Aeraocoustic problems are usually defined on an infinite or semi-infinite domain. The numerical solution of all the discrete equations requires truncation of the computational domain and the imposition of artificial numerical boundary conditions at the truncated domain boundaries. These artificial boundaries must not only ensure non-reflection of waves, but also account for the direction of mean flow with respect to the boundary. Non-reflecting characteristic boundary conditions based on Thompson's technique [22] are used because they are straightforward and easy to apply. There is no obvious reflection observed in the present computations.

For the case of flexible wall discussed later, the vibration amplitude of the flexible boundary is assumed small and this boundary is modeled as a rigid surface with distributed time varying normal velocity perturbations [13].

\section{ILLUSTRATIVE EXAMPLES}

\subsection{The Spinning Vortex Pair}

The sound generated by a spinning vortex pair has an analytical solution [14] and has been investigated numerically by Lee and Koo [15]. This serves as a simple validation case of the present computation code.

The two identical rectilinear vortices, each of a circulation $\Gamma$ and are separated by a distance $2 r_{o}$, undergo co-rotational motions along a circular path with radius $r_{o}$ (Fig. 1). The period of such motion is $T=8 \pi^{2} r_{o}^{2} / \Gamma$, the angular speed $\omega=\Gamma /\left(4 \pi^{2} r_{o}^{2}\right)$ and the rotational Mach number $M_{r}=\Gamma /\left(4 \pi r_{o} c\right)$. The inviscid incompressible flow solution can be expressed in term of a potential function $\phi\left(x_{o}, y_{o}\right)[23]$ :

$$
\phi=\frac{\Gamma}{2 \pi}\left[\tan ^{-1}\left(\frac{y-y_{o}}{x-x_{o}}\right)+\tan ^{-1}\left(\frac{y+y_{o}}{x+x_{o}}\right)\right],
$$

where $\left(x_{o}, y_{o}\right)$ is the vortex location. The hydrodynamic velocity and the hydrodynamic pressure $P$ can be obtained by differentiating Eq. (3) with respect to $(x, y)$ and $t$ respectively:

$$
U=\frac{\partial \phi}{\partial x}, V=\frac{\partial \phi}{\partial y} \text { and } P=p_{o}-\rho_{o} \frac{\partial}{\partial t} \phi\left(x_{o}, y_{o}, t\right)-\frac{1}{2} \rho\left(U^{2}+V^{2}\right)
$$

From the asymptotic expansions by Muller and Obermeier [14], the analytical acoustic pressure variation produced by a spinning vortex pair at a distance $r$ from the vortex system centroid, which is $(0,0)$ in this case, is 


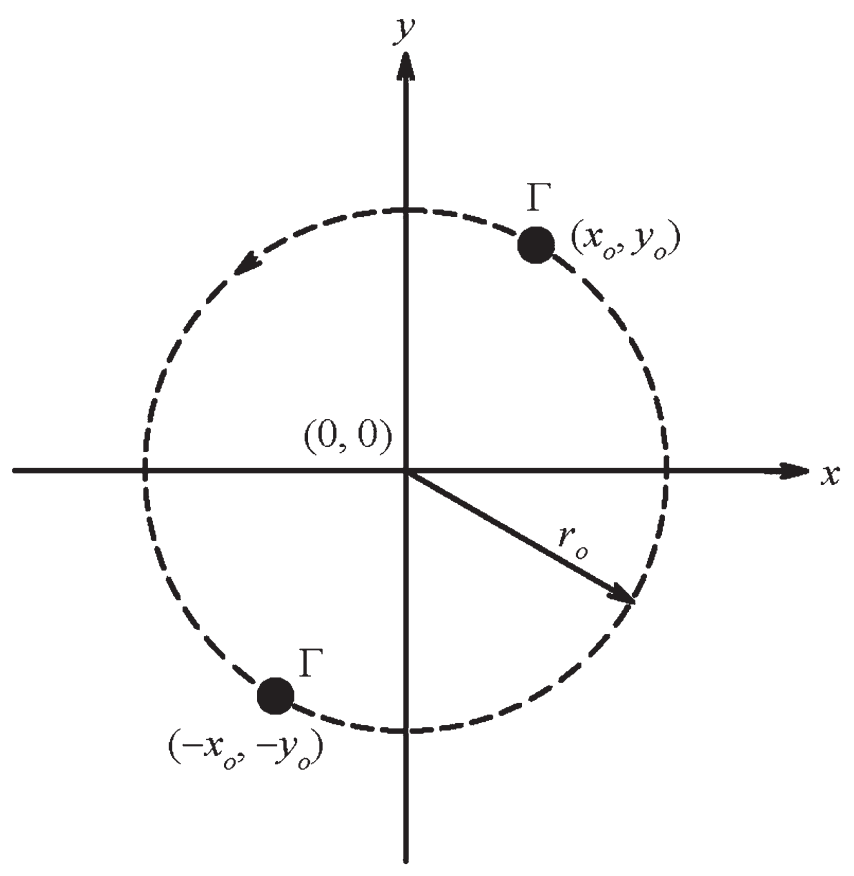

Fig. 1. The spinning vortex pair. ---- : Path of vortex motion; $\bullet$ : vortex of circulation $\Gamma$ (positive).

$$
p^{\prime}(r)=\frac{\rho_{o} \Gamma^{4}}{64 \pi^{3} r_{o}^{4} c^{2}} H_{2}^{(2)}(k r) .
$$

Computations are performed in the domain $[-20<x<20] \times[-20<y<20]$ for $\Gamma /\left(\mathrm{cr}_{o}\right)=1.0$ and $M_{r}=0.0796$ (and thus $k=0.1592$ ). The $[300 \times 300]$ grids are used. The Vatistas vortex-core model $^{24}$ is adopted in order to avoid the singularity at the center of the vortex. At a distance $\sigma$ from the vortex center, the tangential fluid velocity $v_{\theta}$ is given by:

$$
v_{\theta}=\frac{\Gamma}{2 \pi \sqrt{R_{c}^{4}+\sigma^{4}}}
$$

where $R_{c}$ is the core radius.

Figures $2 \mathrm{a}$ and $2 \mathrm{~b}$ present the calculated acoustic pressure contours for the unfiltered and filtered solutions respectively at $t=5$ when the waves are still sufficiently far away from the computational farfield boundaries. A double-spiral pattern of a rotating quadrupole can be observed. The filter is applied to the conserved variables and sequentially in each coordinate direction after each sub-iteration of the implicit Runge-Kutta scheme. The results obtained without a filter show the appearance of high-frequency oscillation and instability as the wave propagates outward in Fig. 2(a). When the high-order filter is employed, these unwanted oscillations are completely eliminated. Figure 2c illustrates the acoustic pressure contours at $t=20$ (filter applied). A comparison of the acoustic pressure profile at this moment along a radial line from the center to the lower-right corner of the computational domain with the theoretical result is shown in Fig. 3. Very good agreement has been reached between the numerical results and the analytical solution. 


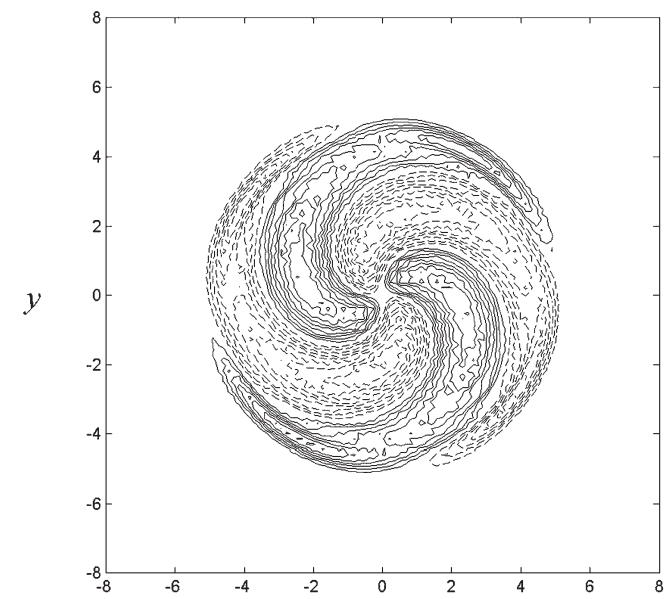

(a)

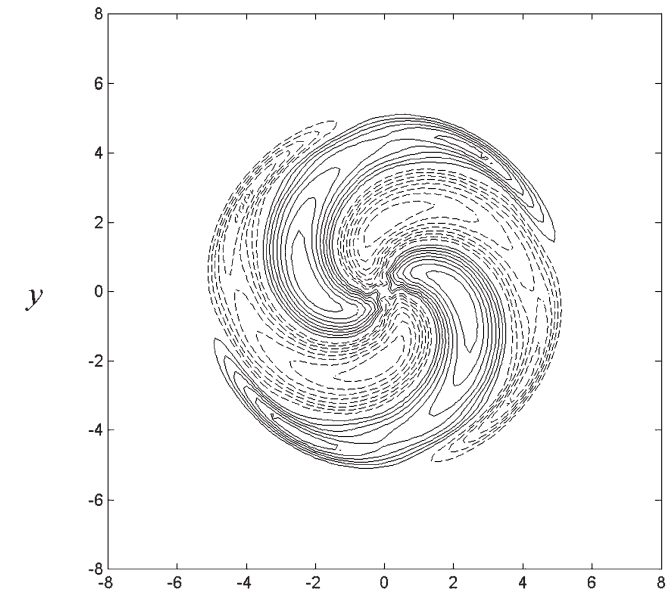

(b)

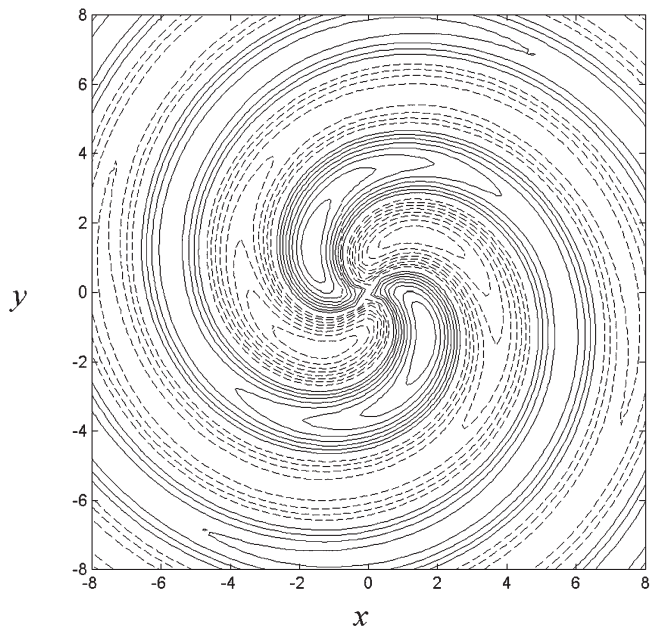

(c)

Fig. 2. Rotating quadrupole produced by the spinning vortex pair (a) $t=5$ without filter; (b) $t=5$ with filter; (c) $t=20$ with filter. $\frac{-}{-}$ : Positive contour; ---- : negative contour. Contour levels: $\pm 2.5 \times 10^{-5}$; $\pm 5 \times 10^{-5} ; \pm 7.5 \times 10^{-5} ; \pm 1 \times 10^{-4} ; \pm 1.25 \times 10^{-4} ; \pm 1.5 \times 10^{-4} ; \pm 1.75 \times 10^{-4} ; \pm 2 \times 10^{-4}$. 


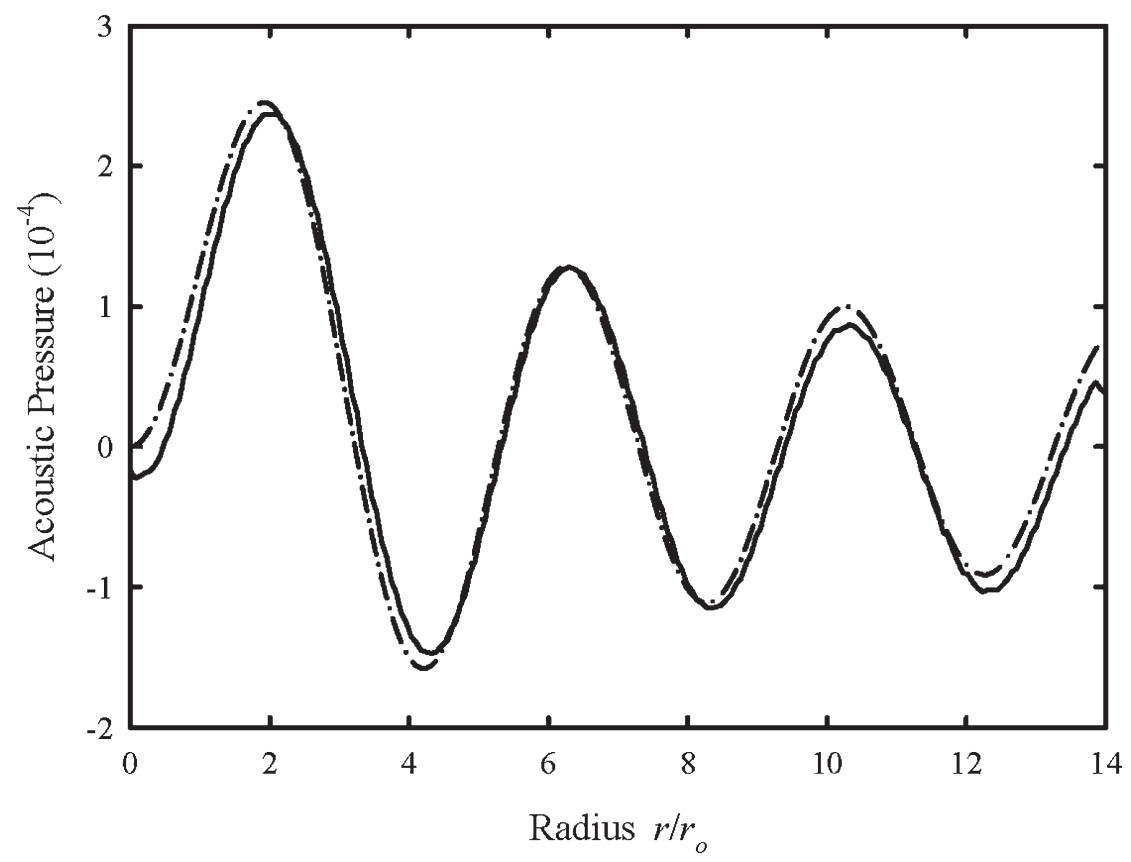

Fig. 3. Radial variation of acoustic pressure along a line making $-45^{\circ}$ with the $x$-axis at $t=20$. Present result; $-\cdot--$ : analytical solution. [17]

\subsection{Vortex Induced Flexible Wall Vibration}

Figure 4 illustrates the schematic of the vortex induced flexible wall vibration model, which is an extract from Tang et al. [13]. A vortex of strength (circulation) $\Gamma$ initially located far upstream of a flexible boundary of length $L$ at a distance $d$ above a rigid plane is considered. The flexible boundary is at rest initially. The vortex motion creates an unsteady fluid pressure on this boundary, causing it to vibrate and this vibration eventually gives rise to a fluctuating velocity field, which affects the motion of the vortex.

As stated in Tang et al. [13], the vibration amplitude $\eta$ of the flexible boundary is assumed small compared to the distance from the vortex center to the plane and this boundary is modeled as a rigid surface with distributed fluctuating velocity $v(x, t)$.

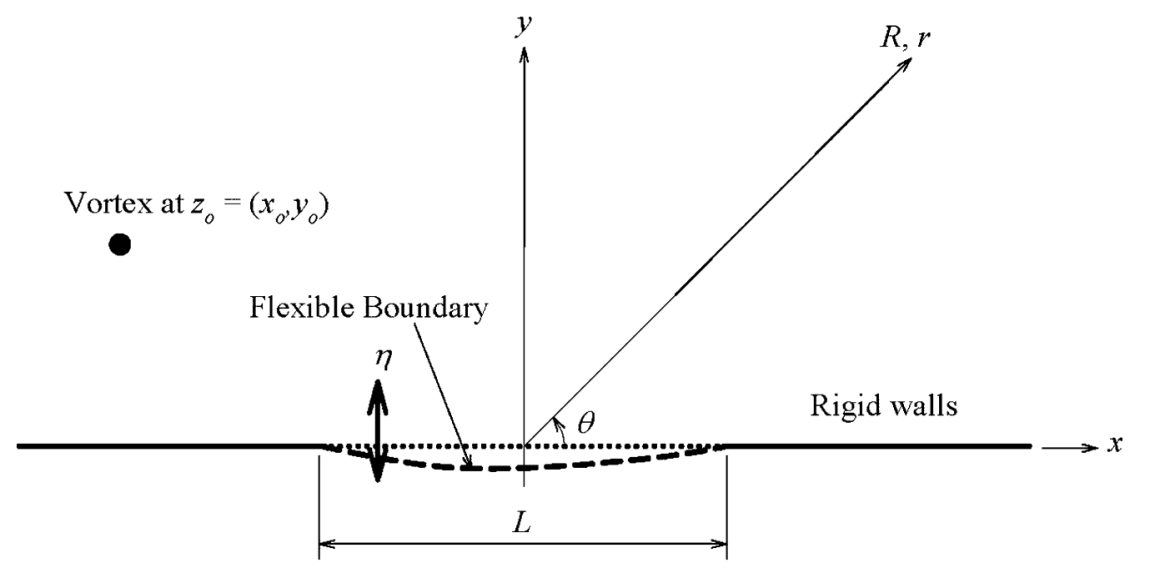

Fig. 4. Schematic of the vortex induced wall vibration model (from Tang et al. [13]). 
The potential function at the position $(x, y)$ in the flow field is a combination of the vortex potential and the flexible boundary vibration potential ${ }^{13}$ :

$$
\phi=\frac{\Gamma}{2 \pi}\left[\tan ^{-1}\left(\frac{y-y_{o}}{x-x_{o}}\right)-\tan ^{-1}\left(\frac{y+y_{o}}{x-x_{o}}\right)\right]+\frac{1}{\pi} \int_{-L / 2}^{L / 2} v \log \sqrt{\left(x-x_{v}\right)^{2}+(y-\eta)^{2}} d x_{v}
$$

where $\left(x_{o}, y_{o}\right)$ is the time varying vortex position and $\left(x_{v}, \eta\right)$ represents the location of a point on the flexible boundary. The induced velocity at the vortex position $\left(x_{o}, y_{o}\right)$ due to the boundary vibration, $V_{i}$, can be determined using potential theory [25] as

$$
V_{i}=\frac{\hat{x}}{\pi} \int_{-L / 2}^{L / 2} \frac{v\left(x_{o}-x\right)}{\left(x_{o}-x\right)^{2}+y_{o}^{2}} d x+\frac{\hat{y}}{\pi} \int_{-L / 2}^{L / 2} \frac{v y_{o}}{\left(x_{o}-x\right)^{2}+y_{o}^{2}} d x
$$

where the caret denotes unit vector in the direction indicated. The vortex velocity $V$, can thus be approximated as:

$$
V=\frac{\Gamma}{4 \pi y_{o}} \hat{x}+V_{i}
$$

The vortex movement path and the movement of the vibrating flexible boundary are obtained from the results of Tang et al. [13] with an in vacuo wave speed along the flexible boundary equals twice the initial speed of the vortex and a normalized damping coefficient of unity. The correspond Mach number of the initial vortex speed $M$ equals 0.05 . The vortex is initially located at $(-4,1)$. Figure 5 illustrates the flight path of the vortex center. The vortex initially moves parallel to the wall. It then moves transversely towards the flexible wall when it is in the

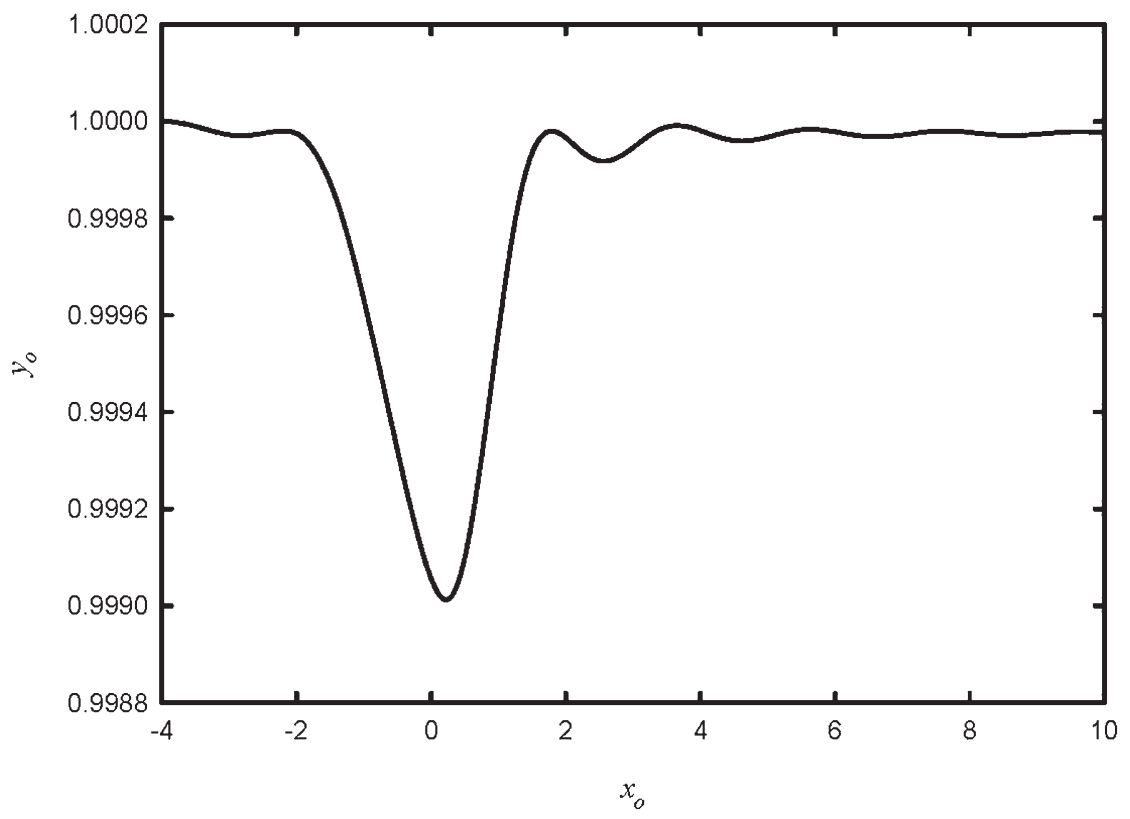

Fig. 5. Vortex flight path for $M=0.05$ (from Tang et al. [13]). 
proximity of the flexible wall. It resumes its original height afterwards. The initial vortex velocity is normalized to 1 .

As described in the spinning vortex case, the hydrodynamic velocity can be obtained by the relationship between velocity and the potential function, while the hydrodynamic pressure can be calculated by using the unsteady Bernoulli's equation. The induced longitudinal and transverse velocities at a position $(x, y)$ due to the boundary vibration are respectively:

$$
U_{\text {flex }}=\frac{1}{\pi} \int_{-L / 2}^{L / 2} \frac{v\left(x-x_{v}\right)}{\left(x-x_{v}\right)^{2}+y^{2}} d x_{v} \text { and } V_{f l e x}=\frac{1}{\pi} \int_{-L / 2}^{L / 2} \frac{v y}{\left(x-x_{v}\right)^{2}+y^{2}} d x_{v} .
$$

Those due to the vortex are

$$
\begin{aligned}
& U_{v o r}=\frac{\Gamma}{2 \pi}\left[\frac{x-x_{o}}{\left(x-x_{o}\right)^{2}+\left(y-y_{o}\right)^{2}}-\frac{x-x_{o}}{\left(x-x_{o}\right)^{2}+\left(y+y_{o}\right)^{2}}\right] \\
& \text { and } V_{v o r}=\frac{\Gamma}{2 \pi}\left[\frac{y-y_{o}}{\left(x-x_{o}\right)^{2}+\left(y-y_{o}\right)^{2}}-\frac{y+y_{o}}{\left(x-x_{o}\right)^{2}+\left(y+y_{o}\right)^{2}}\right] .
\end{aligned}
$$

The corresponding hydrodynamic pressure related to the flowfield induced by the vortex and the flexible wall vibration is respectively:

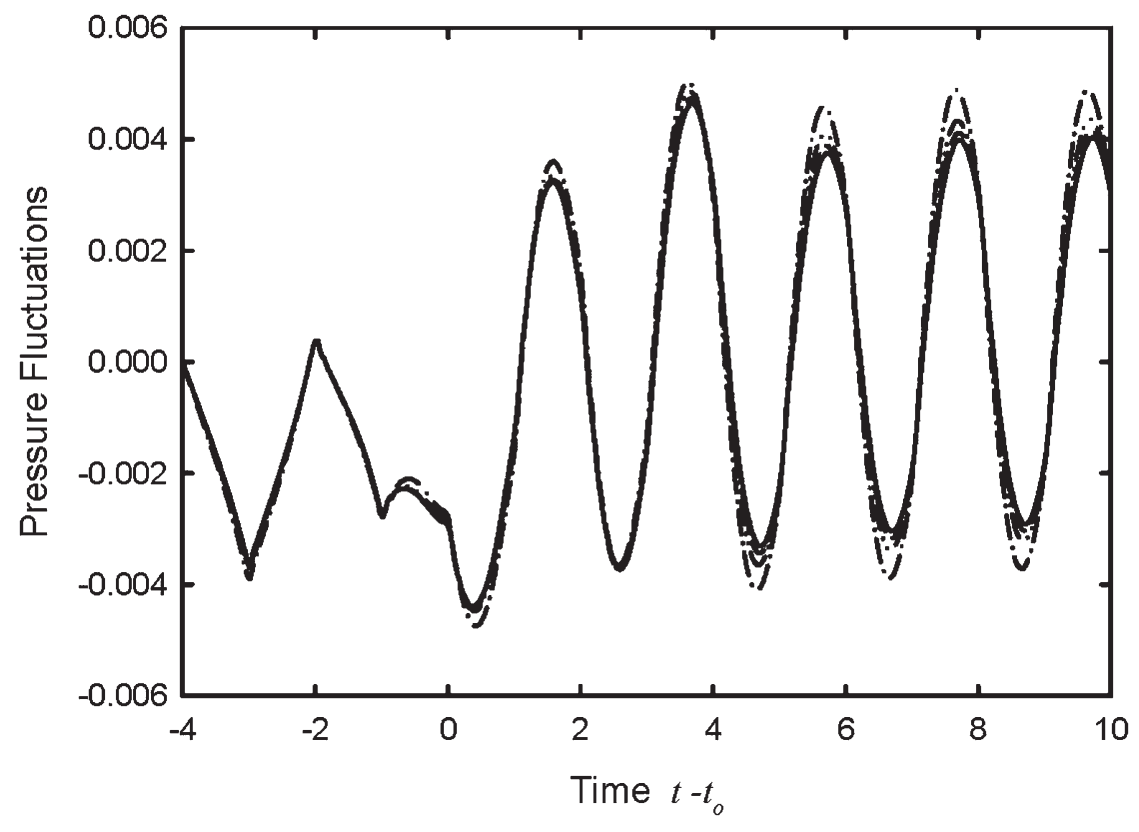

Fig. 6. Effects of grid refinement on the computed acoustic pressure. Test position : $(40,40),-\cdot-$ : $100 \times 100 ;-\cdot-: 200 \times 200 ;----: 300 \times 300 ; \_$_ $400 \times 400$. 


$$
P_{v o r}=p_{o}-\rho_{o} \frac{\partial \phi_{v o r}}{\partial t}-\frac{1}{2} \rho\left(U_{v o i r}^{2}+V_{v o r}^{2}\right) \text { and } P_{\text {flex }}=p_{o}-\rho_{o} \frac{\partial \phi_{\text {flex }}}{\partial t}-\frac{1}{2} \rho\left(U_{\text {flex }}^{2}+V_{\text {flex }}^{2}\right)
$$

The acoustic field induced by the moving vortex can then be obtained by substituting its incompressible hydrodynamic terms into the acoustic disturbance equation, Eq. (2), and so does that from the flexible wall vibration.

A grid refinement study is conducted using the Vatistas vortex model [23]. The computational domain $\Sigma=[-20<M x<20] \times[0<M y<40]$ is covered with $100 \times 100,200 \times 200,300 \times 300$ and $400 \times 400$ uniform numerical grids. The acoustic pressure time history at the point $(40,40)$ is presented in Fig. 6. For the coarse grid of $100 \times 100$, there is an obvious difference between the corresponding results and those of the $400 \times 400$ grid. The acoustic pressure profiles for the $300 \times 300$ and $400 \times 400$ grids almost overlap with each other and the maximum difference is below $1 \%$. Therefore, the $300 \times 300$ uniform numerical grid is used in this study. The time step $\Delta t$ is 0.01 in consistence with that adopted by Tang et al. [1]. The tenth order filter scheme is again applied.

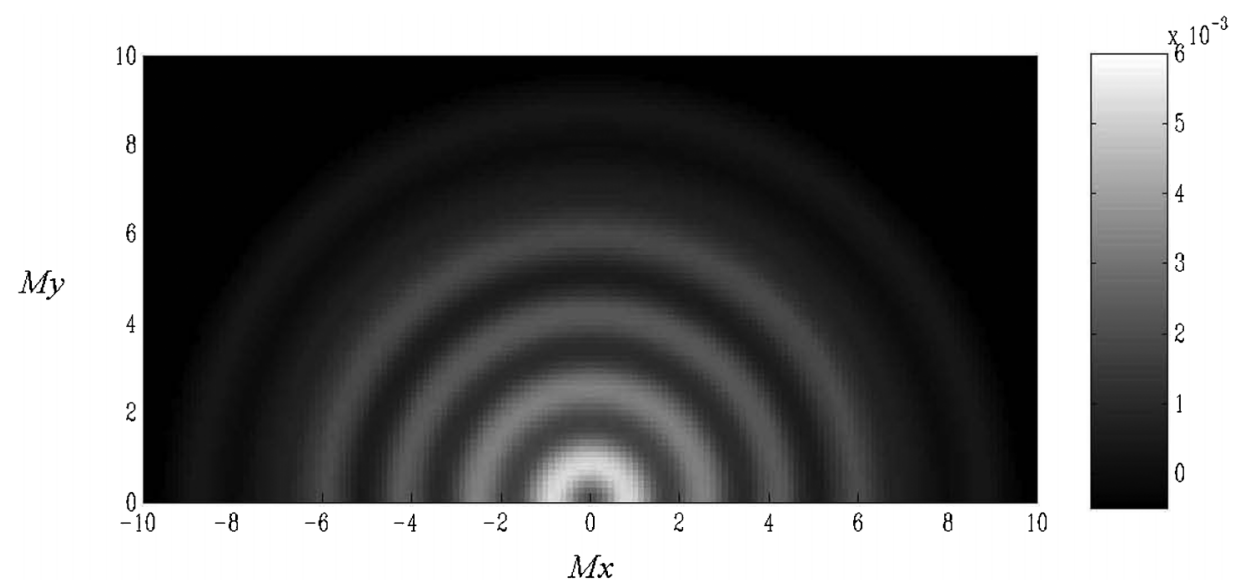

(a)

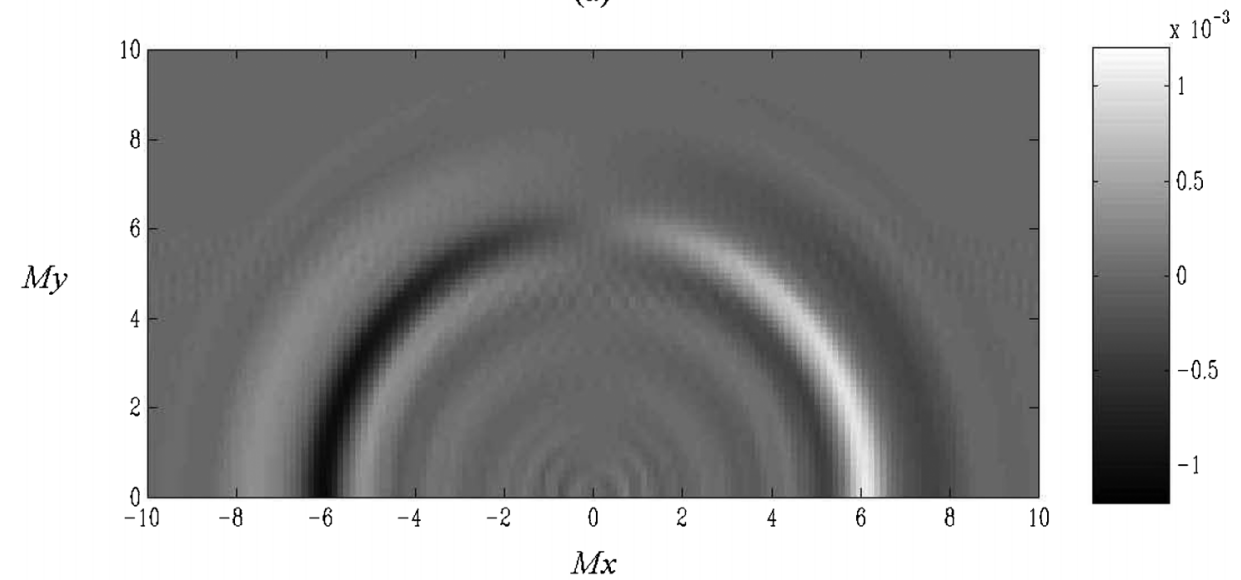

(b)

Fig. 7. Sound fields generated during vortex-flexible boundary interaction at $t-t_{o}=9 . M=0.05$. (a) By flexible boundary vibration; (b) by unsteady vortex motion. 
Figure 7 illustrates the acoustic pressure patterns at $t-t_{o}=9$ when the vortex has propagated far downstream of the flexible wall boundary to $x \sim 8$, where $t_{o}$ denotes the time at which the vortex passes across the plane $x=0$. The vibration of the flexible wall creates a monopole as

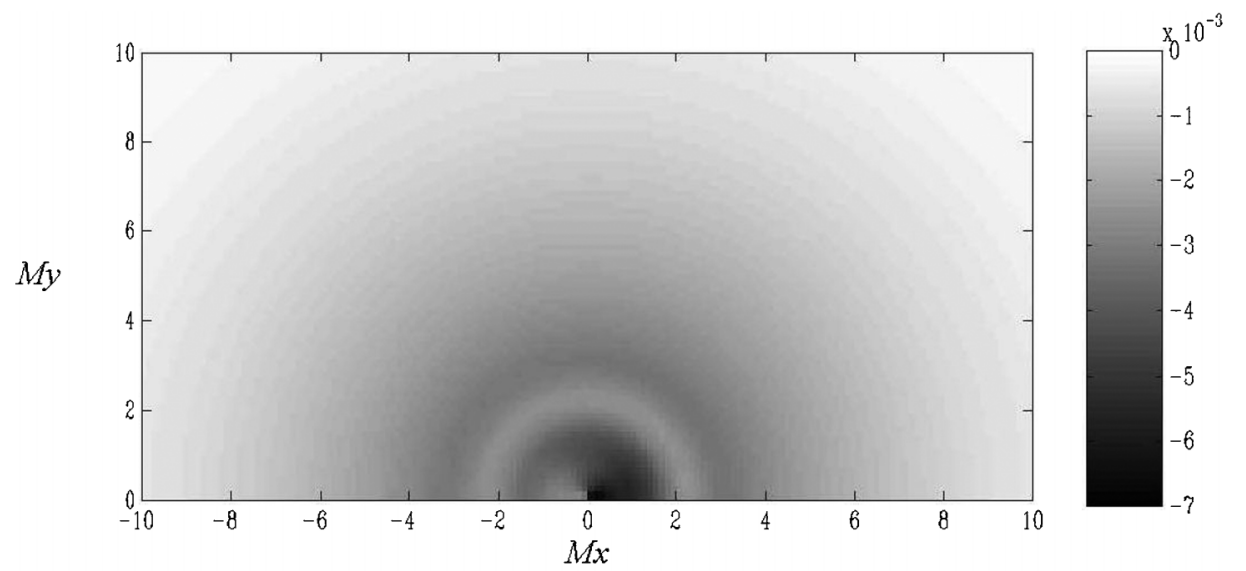

(a)

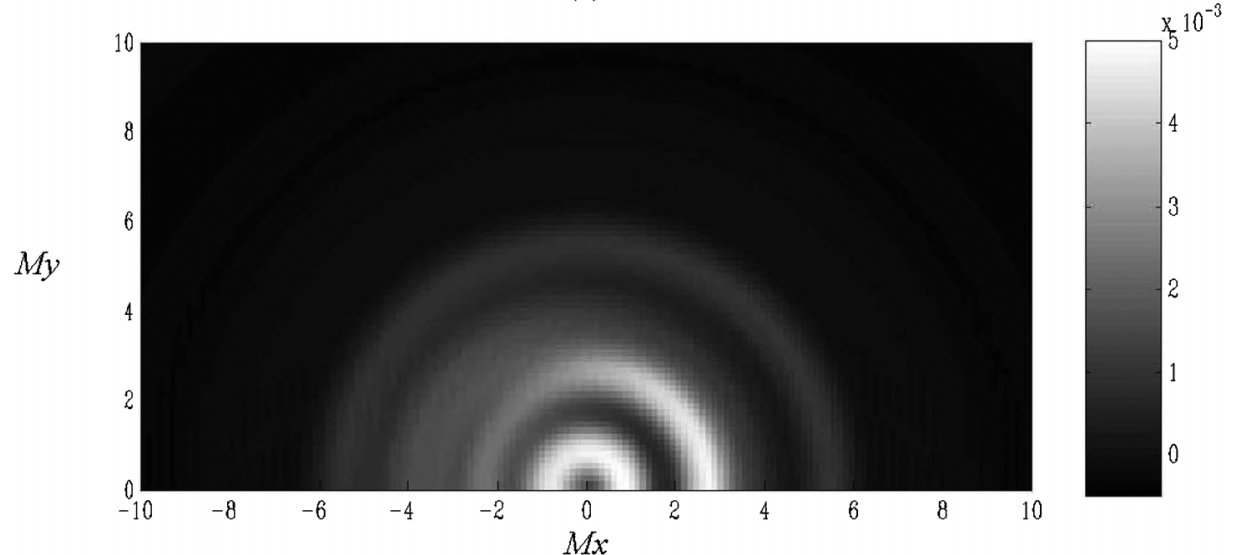

(b)

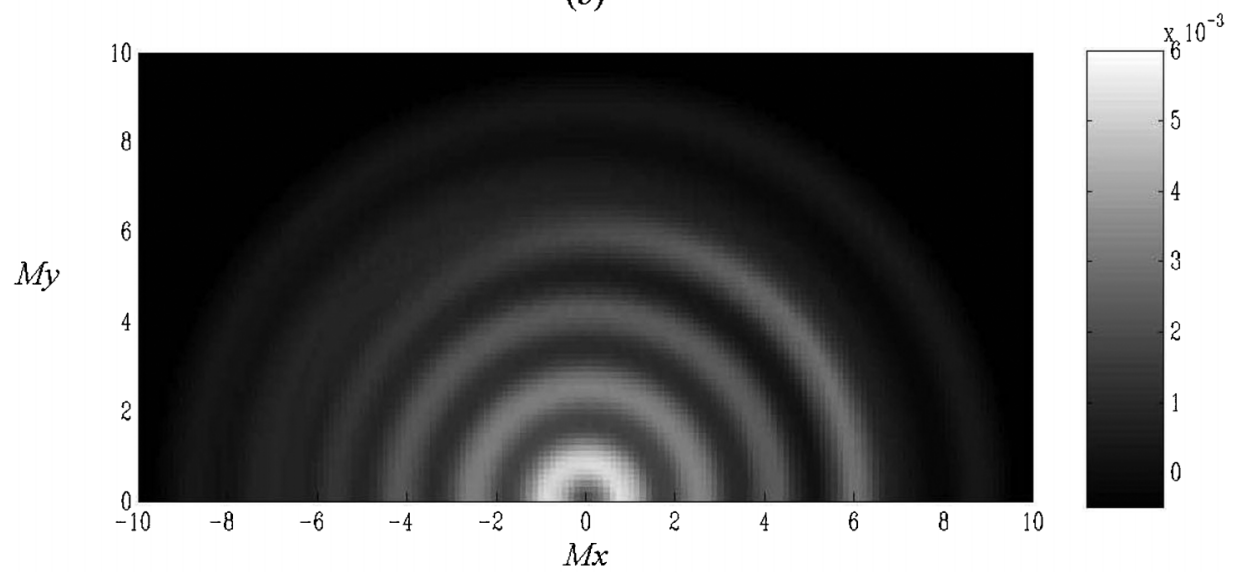

(c)

Fig. 8. Acoustic pressure radiation contours. $M=0.05$. (a) $t-t_{o}=1$ (vortex near to the leading edge of flexible boundary); (b) $t-t_{o}=5$ (vortex at trailing edge of flexible boundary); (c) $t-t_{o}=9$ (vortex far downstream of flexible boundary at $x \sim 8$ ). 
shown in Fig. 7(a) and is still significant even when the vortex is far away from the flexible wall. The unsteady vortex motion generates a dipole but the duration of its generation is relatively short Fig. 7(b). This dipole is generated when the vortex is near to the flexible wall $(|M x| \leq 0.05$ with $M y_{0} \sim 0.05$ ), such that the centre of the radiation appears very near to the origin of the co-ordinate system. The amplitude of the vibration monopole is approximately five times that of the vortex dipole. All these agree with the theoretical calculations of Tang et al. [13].

Figure 8 summarizes the development of the overall sound field. At $t-t_{o}=1$ when the vortex is close to the $x=0$ plane (centerline of the flexible wall), a strongly asymmetric pressure perturbation pattern can be found somewhere near to the flexible wall. This is also the time at which intense interaction between the vortex and the flexible wall is taking place. The very symmetrical radiation in the outer area is from the small flexible wall vibration when the vortex is moving gradually towards the flexible wall. The vortex dipole is therefore significant at the time when the vortex is moving over the flexible wall. Figure 8(b) suggests that the dipole lasts for only around two to three cycles. The whole sound field is eventually dominated by the continuous monopole from the wall vibration Fig. 8(c). One can also observe from Fig. 8 that the radiation directivity is biased to the downstream when the vortex is above the flexible wall, which is also the instant of high transverse vortex acceleration or deceleration. This is also inline with the theoretical deduction of Tang et al. [13].

Figure 9 illustrates the acoustic pressure variation along the $\theta=45^{\circ}$ radial line at $t-t_{o}=12$. Fairly good agreement between the theoretical results of Tang et al. [13] and the present simulation is observed at large radius. It should be noted that the results of Tang et al. [13] are for the far field only and thus discrepancy between the present computed results and that of Tang et al. [13] at small radial distance can be expected. Similar observations can also be made at later instants and along other radial lines and thus are not presented. Similar results are obtained with other combinations of vortex system parameters studied by Tang et al. [13] and

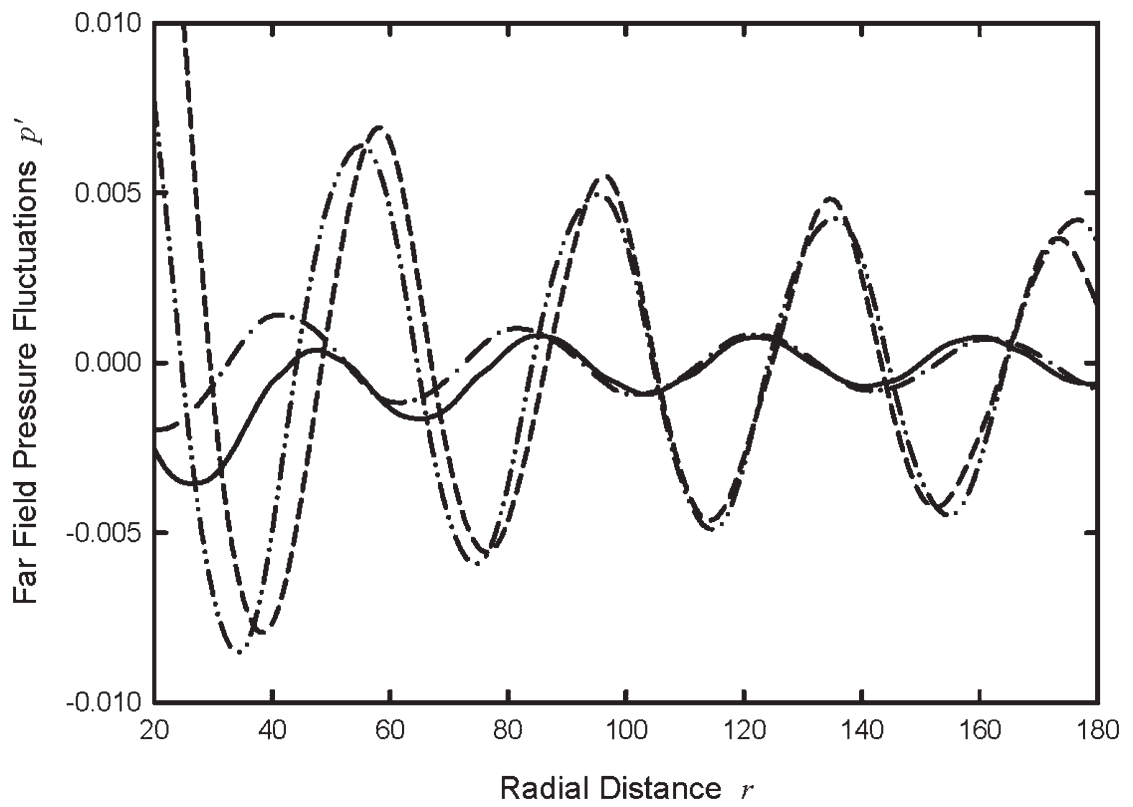

Fig. 9. Radial variation of acoustic pressure along $\theta=45^{\circ}$. For $M=0.05$ at $t-t_{o}=12:-$ : present result; $-\cdot--$ : analytical solution [13]; for $M=0.1$ at $t-t_{o}=20:---$ : present result; $-\cdot \cdot-$ : analytical solution [13]. 
thus they are not presented. The agreement between computed and analytical results in the far field for $M=0.1$ is also very satisfactory.

\section{CONCLUSIONS}

A computational aeroacoustic technique, which splits Euler equations into the hydrodynamic terms and the perturbed acoustic terms is applied. First, the case of the sound generation due to a spinning vortex pair is taken as a benchmark testing case to valid the code. A very good agreement between the numerical simulation and the analytical results is observed.

This method is then applied to the case of sound radiation due to the unsteady interaction between an inviscid vortex and a finite length flexible boundary on an otherwise rigid horizontal plane. Based on the unsteady hydrodynamic information from the known incompressible flow field, the perturbed compressible acoustic terms are calculated. Calculated results are compared with analytical solutions obtained by the method of matched asymptotic expansions. Good agreement between the simulation and the semi-analytical results of Tang et al. [13] is again observed. The present investigation verifies the applicability of the viscous/acoustics splitting approach to flow structure-acoustic interaction. It also implies the possibility of extending the current research to study the complicated interaction between flow turbulence and sound inside a duct silencer for the future development of low self-noise efficient silencing devices.

\section{Acknowledgments}

This study is mainly supported by a grant from Research Grant Council, The Hong Kong Special Administration Region Government, Hong Kong, China (Project no. PolyU5266/05E). The minor financial support from the Research Committee, The Hong Kong Polytechnic University (Project no. G-YD59) is also appreciated.

\section{REFERENCES}

1. Lighthill, M.J., "On sound generation aerodynamically I. General theory," Proceedings of the Royal Society A, Vol. 211, pp. 564-587, 1952.

2. Ffowcs Williams, J.E., Hawkings, D.L., "Sound generated by turbulence and surfaces in arbitrary motion," Philosophical Transactions of the Royal Society of London Series A, Vol. 264, pp. 321-342, 1969.

3. Powell, A., "Theory of vortex sound," Jounal of the Acoustical Society of America, Vol. 36, pp. 177-195, 1964,

4. Möhring, W., "On vortex sound at low Mach number," Journal of Fluid Mechanics, Vol. 85, pp. 685-691, 1978.

5. Shen, W.Z., Sørensen, J. N., "Aeroacoustic modeling of low-speed flows," Theoretical and Computational Fluid Dynamics, Vol. 13, pp. 271-289, 1999.

6. Shen, W.Z., Michelsen, J.A., Sørensen, J.N., "A collocated grid finite volume method for aeroacoustic computations of low-speed flows," Journal of Computational Physics, Vol. 196, pp. 348-366, 2004.

7. Inoue, O., Hatakeyama, N., "Sound generation by a two-dimensional circular cylinder in a uniform flow," Journal of Fluid Mechanics, Vol. 471, pp. 285-314, 2002.

8. Hardin, J., Pope, D. S., "A new technique for aerodynamic noise calculation," Proceedings of the Deutsche Gesellschaft Fur Luft- und (DGLR)/American Institute of Aeronautics and 
Astronautics $14^{\text {th }}$ Aeroacoustics Conference, Aachen, Germany, May 11-14, Vol. 1 (A93-19126 05-71), pp. 448-456, 1992.

9. Shen, W.Z., Sørensen, J.N., "Comment on the aeroacoustic formulation of Hardin and Pope," American Institute of Aeronautics and Astronautics Journal, Vol. 37, pp. 141-143, 1999.

10. Bally, C., Bogery, C. and Juve, D., "Computation of the flow noise using source terms in linearized Euler's equations," American Institute of Aeronautics and Astronautics Journal, Vol. 40, pp. 235-243, 2002.

11. Seo, J.-H. and Moon, Y.J., "Perturbed compression equations for aeroacoustic noise prediction at low Mach numbers," American Institute of Aeronautics and Astronautics, Vol. 43, pp. 17161724, 2005.

12. Ewert, R. and Schröder, W., "Acoustic perturbation equations based on flow decomposition via source filtering," Journal of Computational Physics, Vol. 188, pp. 365-398, 2003.

13. Tang, S.K., Leung, R.C.K., So, R.M.C., Lam, K.M., "Acoustic radiation by vortex induced flexible wall vibration," Journal of the Acoustical Society of America, Vol. 118, pp.2182-2189, 2005.

14. Müller, E.A. and Obermeier, F., "The spinning vortices as a source of sound," Fluids Dynamics of Rotor and Fan Supported Aircraft at Subsonic Speeds, Vol. 22, pp. 1-8, 1967.

15. Lee, D.J. and Koo, S.O., "Numerical study of sound generation due to a spinning vortex pair," American Institute of Aeronautics and Astronautics Journal, Vol. 33, pp. 20-26, 1995.

16. Tam, C.K.W., "Computational aeroacoustics : Issues and methods," American Institute of Aeronautics and Astronautics Journal, Vol. 33, pp. 1788-1796, 1995.

17. Lele, S.K., "Compact finite difference schemes with spectral-like resolution," Journal of Computational Physics, Vol. 103, pp. 16-42, 1992.

18. Visbal, M.R. and Gaitonde, D.V., "On the use of higher-order finite-difference schemes on curvilinear and deforming meshes," Journal of Computational Physics, Vol. 181, pp. 155-185, 2002.

19. Gaitonde, D.V., Visbal, M.R., "Further Development of a Navier-Stokes solution Procedure based on higher-order formulas," American Institute of Aeronautics and Astronautics Paper 990557.

20. Williamson, J., "Low storage Runge-Kutta schemes," Journal of Computational Physics, Vol. 35, pp. 48-56, 1980.

21. Wilson, R.V., Demuren, A.O. and Carpenter, M., "High-order compact schemes for numerical simulation of incompressible flows," Institute for Computer Applications in Science and Engineering Report No. 98-13.

22. Thompson, K. W., "Time dependent boundary conditions for hyperbolic systems," Journal of Computational Physics, Vol. 68, pp. 1-24, 1987.

23. Yuan, S. W., Foundation of fluid mechanics, Prentice-Hall, New Jersey, 1970.

24. Vatistas, G.H., "New model for intense self-similar vortices," Journal of Propulsion and Power, Vol. 14, pp. 462-469, 1998.

25. Vallentine, H.R., Applied Hydrodynamics, Butterworth, London, 1969. 


\section{APPENDIX}

\section{The dynamic equations for disturbance components (Eq. 2)}

The non-linear Euler equations in Cartesian coordinates yield:

$$
\begin{aligned}
& \frac{\partial \rho}{\partial t}+\frac{\partial\left(\rho u_{i}\right)}{\partial x_{i}}=0 \\
& \frac{\partial\left(\rho u_{i}\right)}{\partial t}+\frac{\partial\left(\rho u_{i} u_{j}+p_{i j}\right)}{\partial x_{j}}=0 \\
& \frac{\partial p}{\partial t}=c^{2} \frac{\partial \rho}{\partial t}
\end{aligned}
$$

Decomposing the compressible solution as the sum of a mean and an acoustic disturbance :

$$
u_{i}=U_{i}+u_{i}^{\prime}, p=P+p^{\prime}, \rho=\rho_{o}+\rho^{\prime},
$$

it can be shown by substituting (A-1) into (A-2) that

$$
\begin{aligned}
& \frac{\partial\left(\rho^{\prime}+\rho_{o}\right)}{\partial t}+\frac{\partial\left(\rho^{\prime}+\rho_{o}\right)\left(U_{i}+u_{i}^{\prime}\right)}{\partial x_{i}}=0 \\
& \frac{\partial\left(\rho^{\prime}+\rho_{o}\right)\left(U_{i}+u_{i}^{\prime}\right)}{\partial t}+\frac{\partial\left(\left(\rho^{\prime}+\rho_{o}\right)\left(U_{i}+u_{i}^{\prime}\right)\left(U_{j}+u_{j}^{\prime}\right)+p_{i j}\right)}{\partial x_{j}}=0 \\
& \frac{\partial\left(P+p^{\prime}\right)}{\partial t}=c^{2} \frac{\partial\left(\rho^{\prime}+\rho_{o}\right)}{\partial t}
\end{aligned}
$$

One obtains by subtracting further the incompressible mean flow equations from Eq. A-3 :

$$
\begin{aligned}
& \frac{\partial \rho^{\prime}}{\partial t}+\frac{\partial\left(\left(\rho^{\prime}+\rho_{o}\right) u_{i}^{\prime}+\rho^{\prime} U_{i}\right)}{\partial x_{i}}=0 \\
& \frac{\partial\left(\left(\rho^{\prime}+\rho_{o}\right) u_{i}^{\prime}+\rho^{\prime} U_{i}\right)}{\partial t}+\frac{\partial\left(\left(\left(\rho^{\prime}+\rho_{o}\right) u_{i}^{\prime}+\rho^{\prime} U_{i}\right)\left(U_{j}+u_{j}^{\prime}\right)+\rho_{o} U_{i} u_{j}^{\prime}+p_{i j}^{\prime}\right)}{\partial x_{j}}=0 \\
& \frac{\partial p^{\prime}}{\partial t}-c^{2} \frac{\partial \rho^{\prime}}{\partial t}=-\frac{\partial P}{\partial t}
\end{aligned}
$$


Defining $f_{i}=\rho u_{i}^{\prime}+\rho^{\prime} U_{i}$, Eq. A-4 turns out to be:

$$
\begin{aligned}
& \frac{\partial \rho^{\prime}}{\partial t}+\frac{\partial f_{i}}{\partial x_{i}}=0 \\
& \frac{\partial f_{i}}{\partial t}+\frac{\partial}{\partial x_{j}}\left[p^{\prime} \delta_{i j}+f_{i}\left(U_{j}+u_{j}^{\prime}\right)+\rho_{o} U_{i} u_{j}^{\prime}\right]=0 \\
& \frac{\partial p^{\prime}}{\partial t}-c^{2} \frac{\partial \rho^{\prime}}{\partial t}=-\frac{\partial P}{\partial t}
\end{aligned}
$$

which is Eq. 2. 